\title{
Peptide substrate screening for the diagnosis of SARS-CoV-2 using fluorescence resonance energy transfer (FRET) assay
}

\author{
Hani A. Alhadrami ${ }^{1,2,3} \cdot$ Ahmed M. Hassan $^{2} \cdot$ Raja Chinnappan $^{4} \cdot$ Hind Al-Hadrami $^{5} \cdot$ Wesam H. Abdulaal ${ }^{6}$. \\ Esam I. Azhar ${ }^{1,2} \cdot$ Mohammed Zourob $^{4}$ (i)
}

Received: 4 January 2021 / Accepted: 18 February 2021 / Published online: 25 March 2021

(C) The Author(s), under exclusive licence to Springer-Verlag GmbH Austria, part of Springer Nature 2021

\begin{abstract}
The novel corona (SARS-CoV-2) virus causes a global pandemic, which motivates researchers to develop reliable and effective methods for screening and detection of SARS-CoV-2. Though there are several methods available for the diagnosis of SARSCoV-2 such as RT-PCR and ELSIA, nevertheless, these methods are time-consuming and may not apply at the point of care. In this study, we have developed a specific, sensitive, quantitative and fast detection method for SARS-CoV-2 by fluorescence resonance energy transfer (FRET) assay. The total extracellular protease proteolytic activity from the virus has been used as the biomarker. The specific peptide sequences from the library of 115 dipeptides were identified via changes in the fluorescence signal. The fluorogenic dipeptide substrates have the fluorophore and a quencher at the $\mathrm{N}$ - and the $\mathrm{C}$ - terminals, respectively. When the protease hydrolyzes the peptide bond between the two specific amino acids, it leads to a significant increase in the fluorescence signals. The specific fluorogenic peptide (H-d) produces a high fluorescence signal. A calibration plot was obtained from the changes in the fluorescence intensity against the different concentrations of the viral protease. The lowest limit of detection of this method was $9.7 \pm 3 \mathrm{pfu} / \mathrm{mL}$. The cross-reactivity of the SARS-CoV-2-specific peptide was tested against the MERS-CoV which does not affect the fluorescence signal. A significant change in the fluorescence signal with patient samples indicates that this FRET-based assay might be applied for the diagnosis of SARS-CoV-2 patients.
\end{abstract}

Keywords SARS-CoV-2 detection · COVID-19 FRET substrate · Detection of Covid-19 · Fluorogenic peptide sequences

\section{Introduction}

The outbreak of coronavirus disease 2019 (COVID-19) caused by the severe acute respiratory syndrome coronavirus

Hani A. Alhadrami and Raja Chinnappan contributed equally to this work.

Hani A. Alhadrami

hanialhadrami@kau.edu.sa

$\triangle$ Esam I. Azhar

eazhar@kau.edu.sa

Mohammed Zourob

mzourob@alfaisal.edu

1 Department of Medical Laboratory Technology, Faculty of Applied Medical Sciences, King Abdulaziz University, P. O. Box 80402, Jeddah 21589, Saudi Arabia

2 Special Infectious Agent Unit, King Fahd Medical Research Centre, King Abdulaziz University, P. O. Box 80402, Jeddah 21589, Saudi Arabia
2 (SARS-CoV-2/2019-nCoV) poses a serious threat to global public health and local economies [1]. It causes hundreds of thousands of serious illnesses and human life losses all over the world. Human respiratory tract infections caused by
3 Molecular Diagnostic Lab, King Abdulaziz University Hospital, King Abdulaziz University, P. O. Box 80402, Jeddah 21589, Saudi Arabia

4 Department of Chemistry, Alfaisal University, Al Zahrawi Street, Al Maather, AlTakhassusi Rd, Riyadh 11533, Saudi Arabia

5 Molecular Diagnostic Lab, King Abdullah Medical City Specialist Hospital, Makkah, Saudi Arabia

6 Department of Biochemistry, Faculty of Science, King Abdulaziz University, Jeddah, Saudi Arabia 
coronaviruses such as $\mathrm{HCoV}-229 \mathrm{E}$ and $\mathrm{HCoVOC} 43$ are known since the 1960s [2], and later SARS-CoV-1, HCoVNL63, HCoV-HKU1, and MERS-CoV have been discovered [3]. Severe respiratory illness in humans due to COVID-19 was first reported in December 2019 in China. Since then, $81,187,266$ confirmed cases of infection and $1,772,853$ deaths of 28 December $2020[1,4,5]$. People with chronic diseases such as diabetes, renal failure, chronic lung disease, and immunocompromised patients are at high risk to die due to COVID-19 [4]. The incubation period of COVID-19 varies between 2 and 14 days and is susceptible to all age groups [5]. The virus can be transmitted from human to human and might cause mild illnesses, severe pneumonia, severe respiratory syndrome, and multi-organs failure [6]. Other reported symptoms are fever, anorexia, nausea, vomiting, abdominal pain, diarrhea, and disseminated intercellular coagulation. The WHO and Center for Disease Control (CDC) recommended that COVID-19 cases must be confirmed by RT-PCR via targeting three genes: RdRP gene, $\mathrm{E}$ gene, and $\mathrm{N}$ gene [7]. Different methods have been developed for the diagnosis and detection of COVID-19 such as molecular-based assays, serological based tests, point of care devices, radiology-based tests, and viral cell culture-based methods [8]. The molecular diagnostic assays rely on the identification and amplification of the viral RNA by real-time reverse transcriptasepolymerase chain reaction (rRT-PCR) [9] obtained from the suspected individuals. The WHO and CDC have established a standard protocol with a set of primers that can bind to the specific region of RNA and facilitate the amplification process [10]. When SARS-CoV-2 infects human host cell, it produces a specific antibody against SARS-CoV-2. Enzyme-linked Immunosorbent Assay (ELISA) is used for the qualitative and quantitative detection of SARS-CoV-2 antibody. Chemiluminescent immunoassay (CLIA) is used for the quantitative measurement of SARS-CoV-2 antibodies such as IgG, IgM, and IgA [11]. Western blots have been employed to detect specific SARS-CoV-2 proteins, and Northern blot hybridization has been applied for targeting specific SARS$\mathrm{CoV}-2$ genes [12]. Nucleic acid amplification techniques such as PCR [7, 13], nucleic acid sequence-based amplification (NASBA) [14], next-generation sequencing (NGS) [15], and Lawrence Livermore microbial detection array (LMDA) [16] were developed and optimized for the identification of SARSCoV-2 genome from clinical samples. Although these methods are sensitive, the mutation rate in SARS-CoV-2 genome is high, which may lead to false results. Alternatively, detection of SARS-CoV-2 in short time with low cost has been the focus of this study by the screening of protease using the cleavage of a specific peptide bond from a substrate library. Recently, protease-based nanobiotechnology plays a potential role in the clinical application in the detection and identification of emerging viruses [17-19]. FRET peptides are the potential candidate to follow the enzymatic activity in crude biological fluids and culture media. FRET-based assay is rapid, extremely sensitive, and straightforward. The increased fluorescence signal is directly proportional to the quantity of the protease from SARS-CoV-2.

In this study, we used the proteases as diagnostic markers for the detection of SARS-CoV-2. Several proteases are acting as host receptors for the coronavirus entry including aminopeptidase $\mathrm{N}$ and angiotensin-converting enzyme 2 (hACE2). We developed a simple, low cost, and reliable assay for the detection of the SARS-CoV-2 using the activity of the SARS$\mathrm{CoV}-2$ secreted proteases. We used short peptide containing the cleavage site of the SARS-CoV-2 protease and it was labeled with a fluorophore and a quencher at both ends. The peptide is cleaved in the presence of protease; therefore, the fluorophore and the quencher are separated from each other which leads to high fluorescence. The specific peptide sequence cleaves by the SARS-CoV-2 protease indicates the presence of SARS-CoV-2.

\section{Materials and methods}

\section{Materials}

Vero E6 cells (ATCC® number 1568) were purchased from the American Type Culture Collection (ATCC®). Dulbecco's Eagle medium (DMEM) and fetal bovine serum (FBS), streptomycin and penicillin, HEPES, agarose, paraformaldehyde, crystal violet, PBS, and DMSO were purchased from SigmaAldrich (Gillingham, UK). Dipeptide library was purchased from Pepmic Co Ltd. (Suzhou, China).

\section{Experimental}

\section{Cell line and SARS-CoV-2 propagation}

Vero E6 cells (ATCC® number 1568) were maintained and grown in Dulbecco's Eagle medium (DMEM) contained 10\% fetal bovine serum (FBS) as described before [20]. SARS-CoV2/human/SAU/85791C/2020 (Gene accession number MT630432.1) was isolated from a human nasopharyngeal swab confirmed positive by RT-PCR. IRP number H-02-K-07600520-298 was obtained from the Saudi Ministry of Health to use patient samples. All experiments involved live SARS-CoV2 were performed following the international recommended safety measures and precautions in Biosafety Level 3 Facility at the Special Infectious Agent Unit, King Fahd Medical Research Center, King Abdulaziz University, Saudi Arabia.

SARS-CoV-2 was propagated and titrated using Median Tissue Culture Infectious Dose (TCID50). In brief, SARSCoV-2 was inoculated on 90-95\% confluent Vero E6 cells in a T175 tissue culture flask and incubated at $37{ }^{\circ} \mathrm{C}$ for $1 \mathrm{~h}$ 
in a humidified $5 \% \mathrm{CO}_{2}$ incubator with shaking every $15 \mathrm{~min}$. Then, $25 \mathrm{~mL}$ of viral inoculation medium (DMEM supplemented with $10 \mathrm{mmol} / \mathrm{L}$ HEPES, $1 \%$ streptomycin and penicillin, and $2 \%$ FBS) was used to replace the inoculum. The cells were then incubated at $37{ }^{\circ} \mathrm{C}$ in a humidified $5 \% \mathrm{CO}_{2}$ incubator for $72 \mathrm{~h}$ or until $90 \%$ of the cells illustrated CPE (cytopathic effect). The supernatant was then harvested and centrifuged at $500 \times \mathrm{g}$ for $5 \mathrm{~min}$ at room temperature. Ultimately, SARS-CoV-2 was aliquoted and stored at $-80^{\circ} \mathrm{C}$, and the plaque assay was used to determine the virus titer and TCID50. MERS-CoV was isolated from human nasopharyngeal swab confirmed positive by RT-PCR. The MERS-CoV-positive sample was inoculated on the $95 \%$ confluent Vero E6 cells, and finally, the virus was harvested as described above in the propagation of SARS-CoV-2.

\section{Plaque assay}

Plaque assay was conducted as described previously [21]. In brief, DMEM medium was used to grow $1 \times 10^{5} / \mathrm{mL}$ Vero E6 cells. Every well of six-well tissue culture plates were seeded with $2 \mathrm{~mL}$ Vero E6 cells and incubated at $37^{\circ} \mathrm{C}$ overnight. Serial dilution for each sample (starting from $10^{-1}$ ) was performed in the inoculated DMEM, and $200 \mu \mathrm{L}$ of each dilution were transferred to the Vero E6 cell monolayers and incubated at $37^{\circ} \mathrm{C}$ for $1 \mathrm{~h}$ with shaking every $15 \mathrm{~min}$. Overlay DMEM with $0.8 \%$ agarose was then added to replace the inoculum and incubated at $37^{\circ} \mathrm{C}$ for 3-4 days. Subsequently, the overlay was removed, and 4\% paraformaldehyde was used for 15 min to fix the cells. Crystal violet was used to stain the cells, and plaques were counted to determine SARS-CoV-2 titer as plaque-forming units $(\mathrm{pfu} / \mathrm{mL})$. TCID50 for SARS-CoV-2 was calculated as $3.16 \times 10^{5}$.

\section{Peptide library design}

The peptide library consisted of 115 substrates with two amino acids of the same type or different types including D-amino acids. The library substrates were FRET-based fluorogenic peptides, each peptide had two amino acids of two L-amino acids, or C-terminal L-amino acid and $\mathrm{N}$-terminal D-amino acid, or two D-amino acids. The upper-case letters represent L-amino acids, and the lower-case letter represents D-amino acids. The FITC (fluorescein isothiocyanate) and the dabcyl, [4-((4-(dimethylamino)phenyl)azo)benzoic acid] are the most efficient FRET pairs; therefore, they were introduced at both ends of all the substrates. 6-aminohexanoic acid, Ahx linker was introduced between the FITC fluorophore and the Nterminal amino acids. Lysine was introduced between the Cterminal amino acids and the dabcyl quencher molecule. The combination of different dipeptide substrates has been validated $[22,23]$.

\section{Identification of SARS-CoV-2-specific substrate by FRET assay}

SARS-CoV-2-specific peptide was identified from the library of 115 FRET substrates by high-throughput screening. All the fluorogenic peptides with FITC and the dabcyl were added to the wells of 96 well fluorescence microtiter plate. The proteolytic activity of the COVID-19 protease on the individual substrates was monitored from the changes in the fluorescence of the FITC at its emission maxima. $0.5 \mu \mathrm{L}$ of each peptide from $800 \mu \mathrm{M}$ solution, and $50 \mu \mathrm{l}$ PBS were mixed with $50 \mu \mathrm{l}$ of SARS-CoV-2 total protease (the supernatant of the overnight culture media). Culture media was used as a negative control. The changes in the fluorescence intensity of FITC was monitored every $2 \mathrm{~min}$ for $2.5 \mathrm{~h}$ at $37^{\circ} \mathrm{C}$. The samples were excited at $485 \mathrm{~nm}$ and the emission was recorded at $535 \mathrm{~nm}$. The relative fluorescence unit (RFU) change in each sample with time was calculated by subtracting the sample fluorescence values from the negative control values. The protease activity on the substrate reflects the changes in the RFU per minute (RFU/min). The specific peptide with the highest RFU/m was the most specific to SARS-CoV-2. LHistidine-D-aspartic acid FITC-Ahx-H-d-K(Dabcyl) was found to be the most active peptide in the presence of SARS-CoV-2 protease. FITC-Ahx-H-d-K(Dabcyl) dipeptide was used for further studies.

\section{Real-time fluorescence measurement from the SARS- CoV-2-specific FRET substrate}

The proteolytic activity of SARS-CoV-2 protease on the fluorescence of the FITC-Ahx-H-d-K(Dabcyl) fluorogenic peptides has been studied. A constant amount $(4 \mu \mathrm{M})$ of the H$\mathrm{d}$ peptide in PBS buffer was added to nine different wells of the microtiter plate. Live SARS-CoV-2 viral solution containing $10^{8} \mathrm{pfu} / \mathrm{mL}$ to $10^{1} \mathrm{pfu} / \mathrm{mL}$ was added to each well containing the specific peptide. The changes in the fluorescence were monitored at $535 \mathrm{~nm}$ with the excitation at $495 \mathrm{~nm}$. The fluorescence of FITC in each well was recorded every 2 min for $3 \mathrm{~h}$. The well with the only dipeptide without the live virus was considered as a negative control. The fluorescence values were obtained by subtracting negative control values from the sample values. The raw plot of fluorescence signal change against time for individual samples is presented in Fig. 1a. The percentage change was calculated by $\left(\mathrm{F}-\mathrm{F}_{0} / \mathrm{F}_{0}\right) \times 100$. Figure $1 \mathrm{~b}$ represents the exponential fluorescence signal growth of FITC-Ahx-H-d-K(Dabcyl) peptide in the presence of $10^{8} \mathrm{pfu} / \mathrm{mL}$ at different time intervals.

\section{Detection limit and specificity}

The limit of detection (LOD) of a developed assay determines the successful development of a sensor platform. 

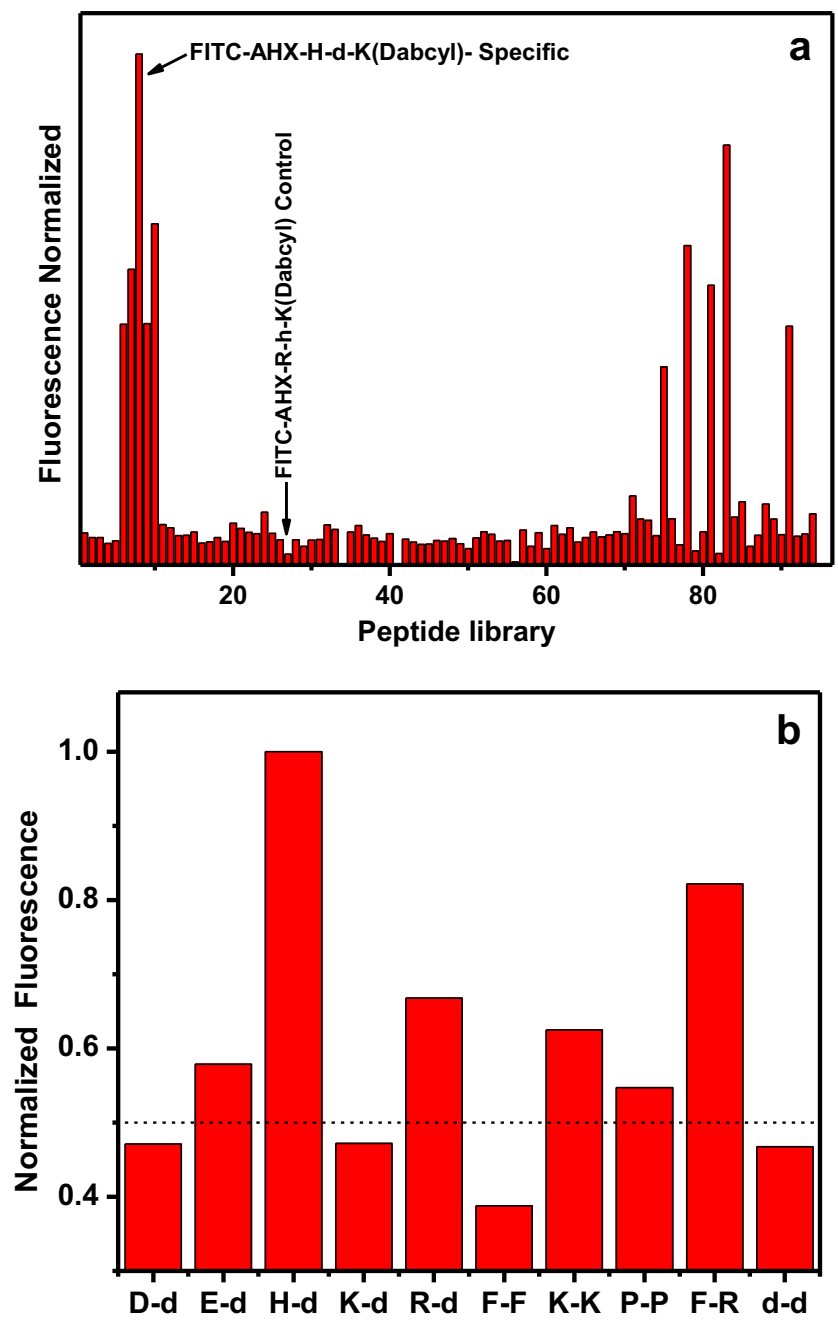

Fig. 1 a The changes in the fluorescence signal of 115 different fluorogenic substrates presence of $10^{8} \mathrm{pfu} / \mathrm{mL}$ at $37^{\circ} \mathrm{C}$. b The FRET substrates with significant increase in the fluorescence signal upon incubation with $10^{8} \mathrm{pfu} / \mathrm{mL}$ of Covid- 19 viral particles at $37^{\circ} \mathrm{C}$. The dotted line represents the threshold fluorescence. The samples were excited at $485 \mathrm{~nm}$ and the fluorescence was observed at $535 \mathrm{~nm}$

The sensitivity of the SARS-COV-2 protease-specific substrate: FITC-Ahx-H-d-K(Dabcyl) was determined from the calibration plot, obtained from the fluorescence of the substrate with different concentrations of the SARS-CoV-2 live virus in the dynamic range of $10^{1}-10^{8} \mathrm{pfu} / \mathrm{mL}$. Each sample with variable concentrations of the protease and their respective fluorescence intensities are represented as the linear plot in Fig. 4. The specificity of the peptide substrate against the other closely associated virus such as MERS-CoV has been tested under the same conditions. No significant change in the fluorescence intensity is observed with MER-CoV protease (Fig. 5). Changes in the fluorescence signal have been observed in the presence of SARS-CoV-2-positive patient sample, and the corresponding $\mathrm{pfu} / \mathrm{mL}$ was calculated from the standard calibration plot.
Real-time RT-PCR for SARS-CoV-2 patient samples

PowerChek ${ }^{\mathrm{TM}}$ 2019-nCoV Real-time PCR Kit (Cat No. R6900TD-100 Samples) was used to perform the RT-PCR test for nasopharyngeal swabs collected from SARS-CoV-2infected patients. The kit was purchased from Kogenebiotech Co., Ltd. Republic of Korea, and it was approved by the FDA. The kit was utilized according to the manufacturer's instructions, and it targets the RdRp gene for 2019-nCoV in nasopharyngeal swab and sputum. In brief, ExiPrep ${ }^{\text {TM }} 96$ Lite was used with ExiPrep ${ }^{\mathrm{TM}} 96$ Viral DNA/RNA Kit (BioNEER Corp) to extract SARS-CoV-2 RNA. PowerChek ${ }^{\mathrm{TM}}$ provides a one-step real-time RT-PCR premix with specific primers and probes. PCR mixture was prepared by adding $11 \mu \mathrm{L}$ of RT-PCR Premix, $4 \mu \mathrm{L}$ of each primer/probe mix, and $5 \mu \mathrm{L}$ of RNA sample to reach a total reaction volume of $20 \mu \mathrm{L}$. The realtime RT-PCR reaction was conducted by programing the LightCycler® 480 Instrument II using the following temperature profile: $50{ }^{\circ} \mathrm{C}$ for $30 \mathrm{~min}(1 \mathrm{cycle}), 95^{\circ} \mathrm{C}$ for $10 \mathrm{~min}$ ( 1 cycle), $95{ }^{\circ} \mathrm{C}$ for $15 \mathrm{~s}$ ( 40 cycles), and $60{ }^{\circ} \mathrm{C}$ for $1 \mathrm{~min}$ ( 40 cycles). The fluorescence curves were analyzed on FAM fluorescence detection channel for the RdRp gene-2019-nCoV and JOE (VIC or HEX) for the internal control. The result was considered positive if the corresponding fluorescence accumulation curve crosses the threshold line. Negative, positive, and internal controls were used, and their results must be passed for the run to be accepted.

\section{Results and discussions}

Enzymes can hydrolyze the peptide bond between the naturally occurring amino acids. The composition of the amino acid pair determines the specificity of the substrate for the enzyme of interest. The favorable unique conformation of amino acids and their chemical structure with their side chains between the peptide links are the most important parameters for the hydrolysis of the peptide bond by the enzymes. Under pathological conditions, the proteolytic activity of the enzymes plays a major role in many pathological processes. Therefore, the identification of these enzymes is important for understanding the mechanism and retarding the pathological enzymatic process. Determination of the hydrolysis of the specific peptide bond would lead to the development of therapeutic inhibition of the process by throughput screening. The proteolytic activity of the proteases in pathogens draws attention to develop sensitive and specific methods to detect pathogens. The fluorogenic (FRET) peptides have a fluorescent donor group attached to one of the amino acids that transfer the excited energy to the quencher acceptor group attached to another amino acid in the substrate by FRET processes [24, 25] (Scheme 1). In general, the donor and the acceptor are in very close proximity in the fluorogenic peptides and show low fluorescence due to the internal quenching (Scheme 1, A). 


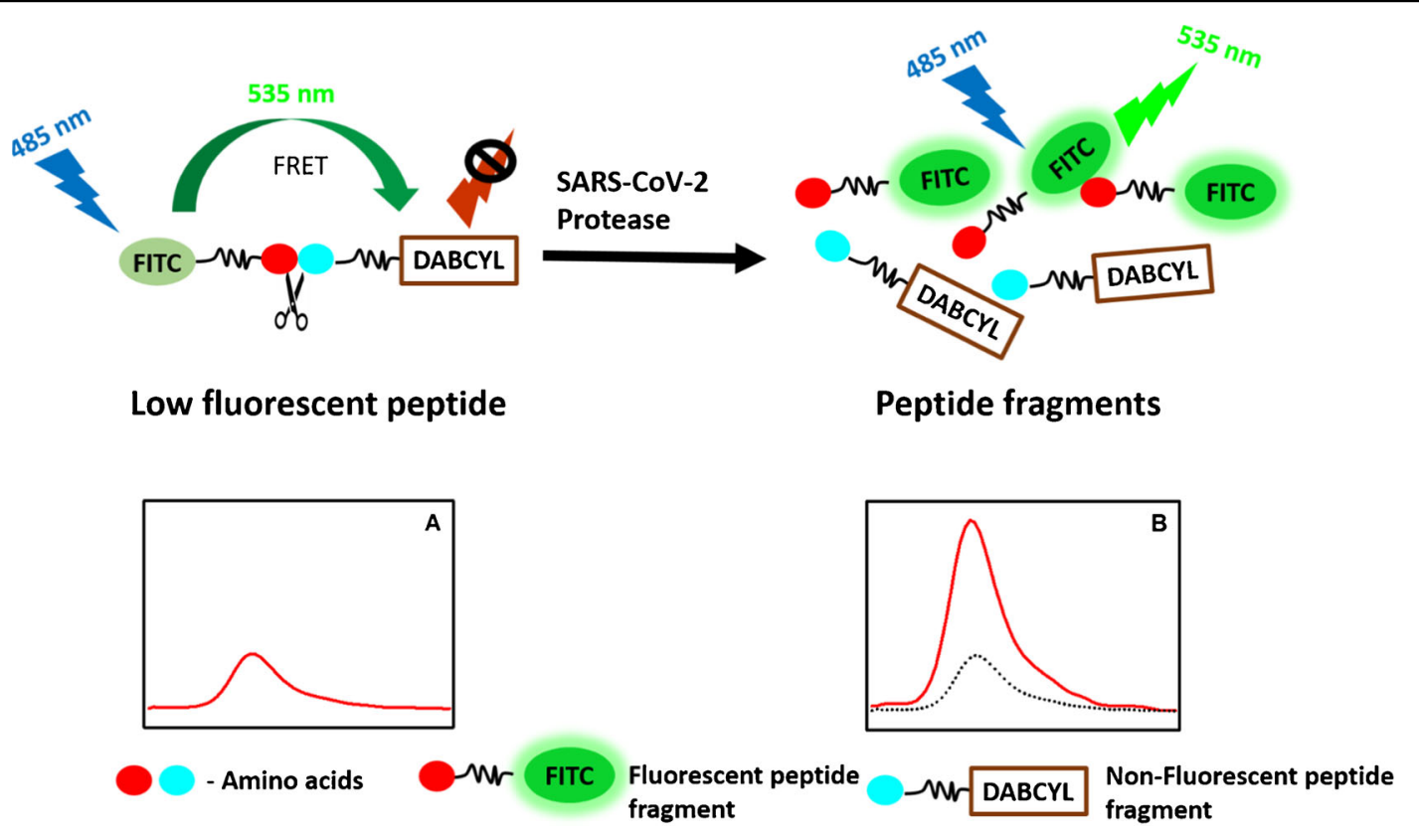

Scheme 1 Schemtaic for the FRET assay used for screening the peptides library and the assay development. (A) Fluorescent molecule and quencher attached to the peptide seqeunce (low fluorescence signal); (B) protease cleaves the peptide seqeunce and separate the fluorecent molecules from the quencher, which results in increase in the fluorescence signal

amino acids which is the only peptide bond that undergoes hydrolysis and induces the fluorescence signal [23]. SARS$\mathrm{CoV}$ and SARS-CoV-2 main protease-specific substrates have been screened by a high-throughput screening method using both natural and unnatural amino acids [19, 29, 30]. In this screening process, the SARS-CoV-2 total protease was incubated with the individual FRET substrate for $2 \mathrm{~h}$ at $37{ }^{\circ} \mathrm{C}$. Change in the fluorescence intensity of each peptide was compared to find the efficient hydrolysis of the peptide bond by SARS-CoV-2 protease on the specific peptide substrate. The fluorescence signal response generated by substrate hydrolysis is illustrated in Fig. 1a. We selected ten substrates that showed considerable fluorescence signal change in the presence of SARS-CoV-2 protease (D-d, E-d, H-d, K-d, R-d, F-F, P-P, F-R, and d-d). The relative fluorescence intensity of the substrates is summarized in Table 1. The highest fluorescence change was observed from FITC-Ahx-H-d$\mathrm{K}$ (Dabcyl dipeptide), and thus, we considered this the relative scale of $100 \%$. FITC-Ahx-F-R-K(Dabcyl) confirmed $82 \%$ increase in the fluorescence intensity compared to $\mathrm{H}-\mathrm{d}$ peptide (Fig. 1b). Interestingly, most of the active peptide has a combination of L- and D-amino acids. More specifically, the Lamino acids at the $\mathrm{N}$-terminal and $\mathrm{D}$-amino acids in the $\mathrm{C}$ terminals with the exception of F-R and d-d. The two highly sensitive peptides, FITC-Ahx-H-d-K(Dabcyl) and FITC-AhxF-R-K(Dabcyl), have the L-amino acids with conjugated cyclic side chains, and both peptides comprise the positive charge in one of the amino acids. The results indicated that SARS-CoV-2-specific dipeptide substrate has either the 
Table 1 Changes in the fluorescence signal of different substrates in the presence of SARS-CoV-2 protease

\begin{tabular}{ll}
\hline FRET substrate & Relative fluorescence \\
\hline FITC-Ahx-H-d-K(Dabcyl) & 100 \\
FITC-Ahx-F-R-K(Dabcyl) & 82 \\
FITC-Ahx-R-d-K(Dabcyl) & 67 \\
FITC-Ahx-K-K-K(Dabcyl) & 62 \\
FITC-Ahx-E-d-K(Dabcyl) & 58 \\
FITC-Ahx-P-P-K(Dabcyl) & 55 \\
FITC-Ahx-K-d-K(Dabcyl) & 47 \\
FITC-Ahx-D-d-K(Dabcyl) & 47 \\
FITC-Ahx-d-d-K(Dabcyl) & 47 \\
\hline
\end{tabular}

combination of both L- and D-amino acids or only L-amino acids with cyclic side chain and/or positive charge in one of the amino acids. More than $50 \%$ of the active peptides have at least one dicarboxylic acid amino acids (aspartic acid or glutamic acid) implies that the presence of dicarboxylic acid amino acids might be one of the favorable factors for SARSCoV-2 protease proteolytic activity. SARS-CoV-2 protease digests the FITC-Ahx-H-d-K(Dabcyl) efficiently and showed maximum fluorescence intensity after $3 \mathrm{~h}$ of incubation at $37^{\circ} \mathrm{C}$. Interestingly, the fluorogenic dipeptide FITC-Ahx-D$\mathrm{d}-\mathrm{K}$ (Dabcyl) and FITC-Ahx-d-d-K(Dabcyl) had the same effect on the cleavage of peptide bond independently of L- or Damino acids (Fig. 1b), which indicates the presence of specific amino acid in the protease binding site and their orientations were important for the peptide bond cleavage in the substrate. The FITC-Ahx-R-h-K(Dabcyl) was very stable in the presence of SARS-CoV-2 protease incubated at $37{ }^{\circ} \mathrm{C}$ and confirmed insignificant increase in the fluorescence intensity after $3 \mathrm{~h}$. Based upon the fluorescence results, we selected FITCAhx-H-d-K(Dabcyl) for further studies, and we used FITCAhx-R-h-K(Dabcyl) as a control.

\section{Proteolytic activity of SRAS-CoV-2 protease on FITC- Ahx-H-d-K(Dabcyl) substrate}

The rate of cleavage for the H-D substrate in the presence of variable concentrations of SARS-CoV-2 protease was monitored when incubated with $10^{1}-10^{8} \mathrm{pfu} / \mathrm{mL}$ of SARS-CoV-2 supernatant. The fluorescence intensity of the FRET substrate increases rapidly in the presence of $10^{8} \mathrm{pfu} / \mathrm{mL}$ of protease (Fig. 2, black curve). The rate of hydrolysis of the FITC-Ahx$\mathrm{H}-\mathrm{d}-\mathrm{K}$ (Dabcyl) peptide reduces with a reduction in the protease concentrations as illustrated in Fig. 2 curves from top to bottom. There was no significant change in the fluorescence signal of the substrate with $10^{1} \mathrm{pfu} / \mathrm{mL}$ of the protease. The fluorescence value is close to the control sample after $3 \mathrm{~h}$ of incubation at $37^{\circ} \mathrm{C}$ (Fig. 2 dotted line). The fluorescence signal of FITC-Ahx-H-d-K(Dabcyl) peptide substrate was
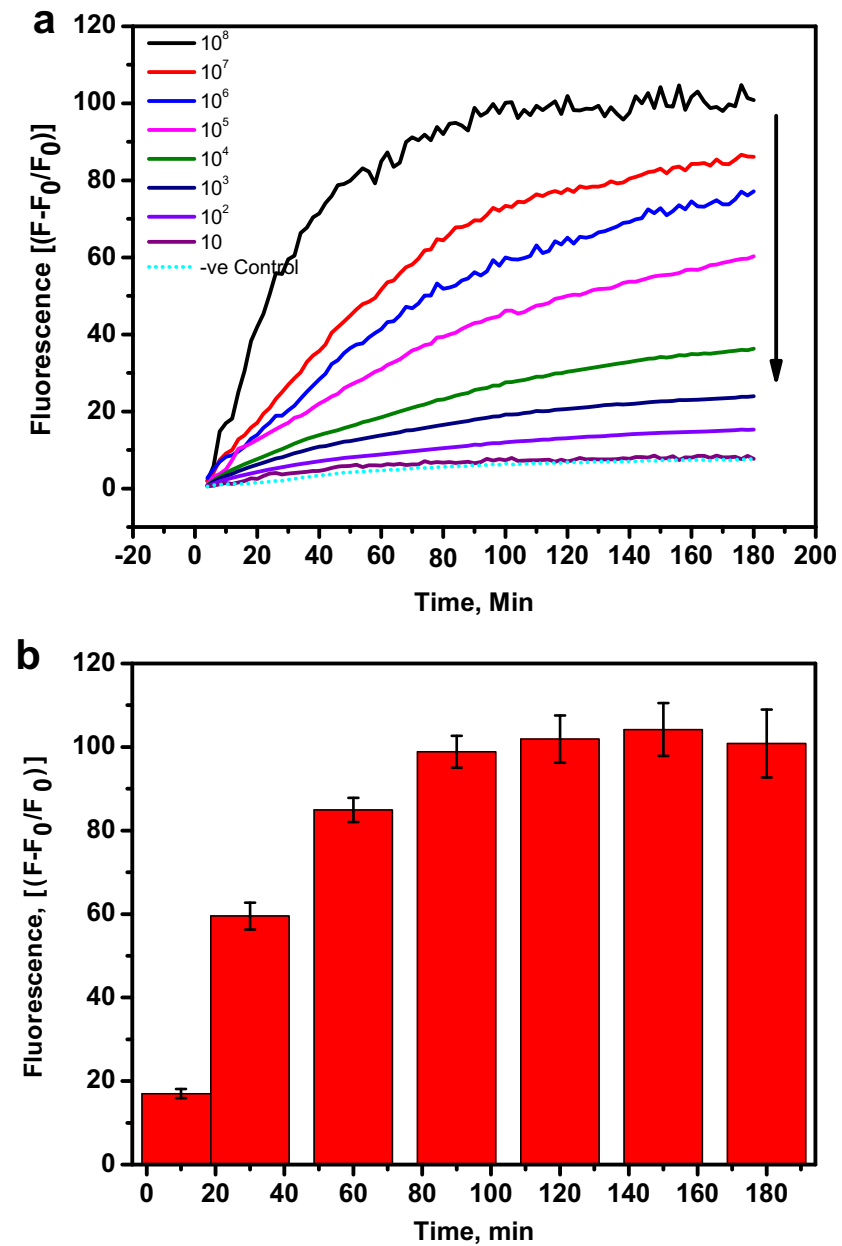

Fig. 2 (a) The real time fluorescence change in FITC-Ahx-H-d(Dabcyl)peptide in the presence of various concentrations of Covid-19 live virus in the dynamic range of $10^{8}-10 \mathrm{pfu} / \mathrm{mL}$ at $37{ }^{\circ} \mathrm{C}$. (b) The increase in the fluorescence signal of FITC-Ahx-H-d-(Dabcyl) incubation with $10^{8} \mathrm{pfu} / \mathrm{mL}$ of Covid- 19 viral particles at $37^{\circ} \mathrm{C}$. The samples were excited at $485 \mathrm{~nm}$ and the fluorescence was observed at $535 \mathrm{~nm}$. The standard errors were calculated from the three different measurements

increasing linearly with time in the presence of $10^{8} \mathrm{pfu} / \mathrm{mL}$ of the protease, indicating that the rate of hydrolysis was very fast in the initial stage of the reaction. The rate decreased over the period of time, and the curve reached a plateau after $1.5 \mathrm{~h}$. When the protease and the FRET substrates mixed in the wells at the early stage, more number of substrates were available for the protease to digest the H-d peptide link in the FITCAhx-H-d-K(Dabcyl) substrate. The protease to substrate ratio decreased with time, and fewer substrate numbers were available for the hydrolysis, and thus, the increase in the fluorescence intensity was not linear anymore. The kinetics curves of the FITC-Ahx-H-d-K(Dabcyl) with different concentrations are illustrated in Fig. 2a. At the initial stage of the reaction, a greater number of FITC-Ahx-H-d-K(Dabcyl) substrates were available for the protease to cleave and the reaction was extremely fast. However, there was less relative fluorescence units (rfu/min) after $30 \mathrm{~min}$ due to the limited number of 
substrates available for the protease to interact with. The changes in the protease amount after the reaction was insignificant due to the high protease concentration in this reaction. Therefore, the rate of the reaction was only dependent on the concentration of the substrate.

\section{SARS-CoV-2 sensing using FRET substrate}

The sensitive detection of SARS-CoV-2 was performed by the hydrolysis of the specific peptide bond between $\mathrm{H}-$ and d-amino acids in FITC-Ahx-H-d-K(Dabcyl) substrate by the total protease. When the substrate was incubated with live SARS-CoV-2 viral particles, the protease produced as a metabolic by-product hence, specifically digest the $\mathrm{H}-\mathrm{d}$ bond rapidly and the FITC donor and the dabcyl acceptor were separated from each other. Therefore, when FITC was excited at $485 \mathrm{~nm}$, the emitted photons were not absorbed by the dabcyl acceptor, because the donor and the acceptor were not in close vicinity and there was no fluorescence quenching. All the emitted photons were monitored as fluorescence emission. The quantitative detection of SARS-CoV-2 was achieved by incubating a constant amount of FRET fluorogenic peptide with different concentrations of SARS-CoV-2 viral protease ranging from 10 to $10^{8} \mathrm{pfu} / \mathrm{mL}$ at $37^{\circ} \mathrm{C}$ for $3 \mathrm{~h}$. Then, the fluorescence of FITC in all the samples is monitored, and the relative change in the fluorescence is represented in Fig. 3. The black and red bars represent the change in the fluorescence intensity after $180 \mathrm{~min}$ and $30 \mathrm{~min}$, respectively. A significant amount of the reaction was completed within $30 \mathrm{~min}$. For example, in the presence of $10^{8} \mathrm{pfu} / \mathrm{mL}$ of SARS-CoV-2, more than $50 \%$ of the reaction was completed within $30 \mathrm{~min}$. Figure 4 illustrates the plot of the endpoint of

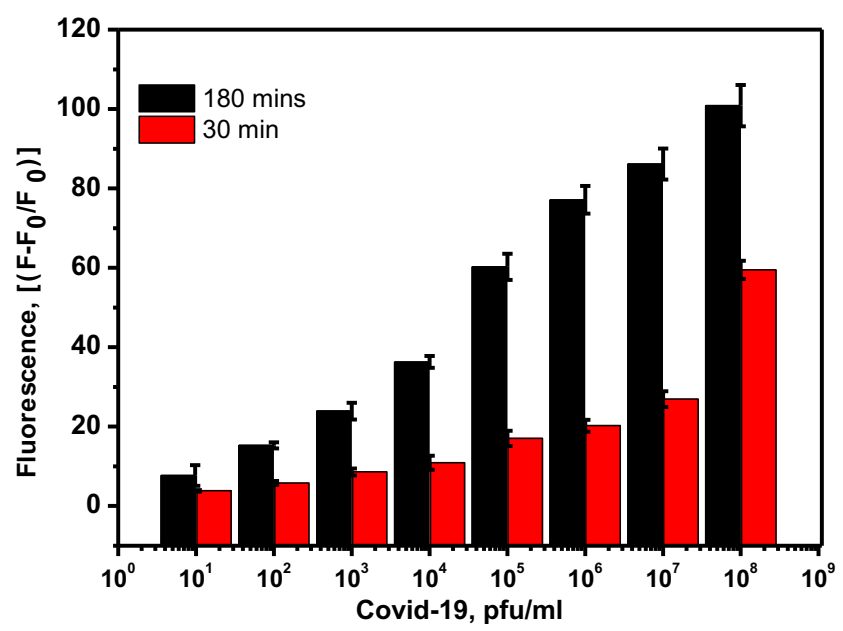

Fig. 3 Changes in the fluorescence of FITC-Ahx-H-d-(Dabcyl) peptide substrate in the presence of various concentrations of Covid-19 live virus $\left(10^{8}-10 \mathrm{pfu} / \mathrm{mL}\right)$ after 30 minutes and 3 hours of incubation at $37^{\circ} \mathrm{C}$. The samples were excited at $485 \mathrm{~nm}$ and the fluorescence was observed at $535 \mathrm{~nm}$. The standard errors were calculated from the three different measurements

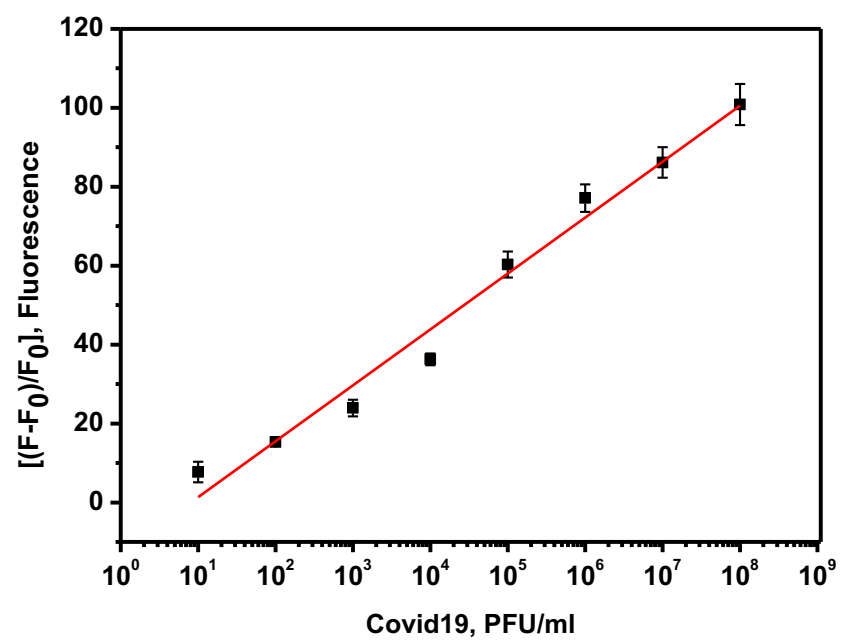

Fig. 4 The plot of change in the fluorescence of FITC-Ahx-H-d(Dabcyl)peptide substrate against the various concentrations of Covid19 live virus $\left(10^{8}-10 \mathrm{pfu} / \mathrm{mL}\right.$ ) after 3 hours of incubation at $37^{\circ} \mathrm{C}$. The samples were excited at $485 \mathrm{~nm}$ and the fluorescence was observed at $535 \mathrm{~nm}$. The standard errors were calculated from the three different measurements

fluorescence values after $3 \mathrm{~h}$ against the respective concentrations of the viral particle. Further, it shows the calibration curve which was obtained by plotting the fluorescence signal change against the logarithm of SARS-CoV-2 particle (protease) concentration (pfu/mL). The minimum SARSCoV-2 sensing ability of FITC-Ahx-H-d-K(Dabcyl), (limit of detection or LOD), was calculated according to the following formula: $3 \mathrm{SD} / \mathrm{m}$, where (SD) is the standard deviation of the fluorescence signal in the absence of SARS-CoV-2 particles and $(\mathrm{m})$ is the slope of the straight line obtained from the linear fitting. The calculated LOD of FITC-Ahx-H-d$\mathrm{K}$ (Dabcyl) substrate was found to be $9 \pm 3 \mathrm{pfu} / \mathrm{mL}$. Our FRET-based results were compared with the RT-PCR method, which is the gold standard for SARS-CoV-2 diagnosis. Table 2 represents the comparison of eight different patient samples (high CT, medium CT, and near the cutoff CT). The samples with rfu of 77 to 100 showed high CT values, rfu of 25 to 60 had medium CT values, and rfu of 25 and less had a

Table 2 Comparison of FRET assay with RT-PCR

\begin{tabular}{lll}
\hline Patient samples & $\begin{array}{l}\text { Fluorescence } \\
\text { (relative percentage) }\end{array}$ & RT-PCR (CT value) \\
\hline Patient 1 & $71-100$ & High \\
Patient 2 & & High \\
Patient 3 & & High \\
Patient 4 & $31-70$ & Medium \\
Patient 5 & & Medium \\
Patient 6 & & Medium \\
Patient 7 & & Weak $(\sim$ Cut off $)$ \\
Patient 8 & $<30$ & Weak $(\sim$ Cut off $)$ \\
\hline
\end{tabular}


Table 3 Comparison of different methods used for the detection of SARS-CoV-2

\begin{tabular}{lllll}
\hline & Method & Sample type & LOD & Reference \\
\hline 1 & RT-PCR & Viral -RNA & 0.91 copies $/ \mathrm{mL}$ & {$[30]$} \\
2 & RT-PCR & RdRp gene & 3.6 copies $/$ reaction & {$[7]$} \\
3 & Field effect transistor & S-protein antibody & 16 copies $/ \mathrm{mL}$ & {$[31]$} \\
4 & RT-LAMP & mRNA & 50 copies $/ \mu \mathrm{L}$ & {$[33]$} \\
5 & RT-q(PCR-LAMP) & mRNA & 5 copies $/$ reaction & {$[34]$} \\
6 & ITP-Crisper & cDNA of mRNA & 10 copies $/ \mu \mathrm{L}$ & {$[35]$} \\
7 & Molecular POC & mRNA & $0.8 \mathrm{pg} / \mathrm{mL}$ & {$[36]$} \\
8 & Electrochemical & Viral antigen & $1 \mathrm{pg} / \mathrm{mL}$ & {$[37]$} \\
9 & Lateral flow immunoassay & IgM/IgG & $0.9 \mathrm{copies} / \mathrm{mL}$ & {$[38]$} \\
\hline
\end{tabular}

weak CT values (within the cutoff values). The results confirmed that our FRET-based assay is comparable with the standard methods such as RT-PCR. Corman et al. developed RT-PCR method for the detection of SARS-CoV-2 and quantified the limit of detection in the range of 2 to 12 copies per reaction [7]. Van Kasteren et al. compared the commercially available RT-PCR kits with their in-house developed method for the detection of SARS-CoV-2. The detection limit of the evaluated kits was in the range of 3 to 10 copies $/ \mathrm{mL}$; however, their in-house PCR method has the LOD of 0.91 copies $/ \mathrm{mL}$ [30]. A field-effect transistor (FET)-based biosensor has been developed for the sensitive detection of SARS-CoV-2 in clinical sample. The sensing platform was fabricated by coating graphene sheets of the FET with anti-SARS-CoV-2 spike protein antibody. The sensor was tested for antigen protein, cultured virus, and specimen from the SARS-CoV-2-positive patients. The sensor was able to detect as low as $16 \mathrm{pfu} / \mathrm{mL}$ [31]. Two high-affinity aptamers against the receptor-binding domain (RBD) of SARS-CoV-2 spike glycoprotein have been selected with the affinity of $5.8 \mathrm{nM}$ and $19.9 \mathrm{nM}$, respectively. The aptamer-based recognized receptor is an ideal tool for the development of sensitive and selective aptasensor for the detection of SARS-CoV-2 [32]. Various methods used for the detection of SARS-CoV-2 are compared with our developed assay and summarized in Table 3 . The previously reported RT-PCR method is more sensitive than other methods due to amplification processes. However, the other methods are comparable or less sensitive than the reported values by our FRET assay. Although our developed assay is highly sensitive, it has some limitations. The turbid sample leads to fluorescence scattering; therefore, samples should be clear. The presence of any molecule in the sample absorbs in the region of the FITC emission which reduces the fluorescence intensity due to the fluorescence quenching. The fluorogenic substrates have to be protected from the light as it may undergo photobleaching when exposing to light.

\section{Cross-reactivity of FRET substrate}

The selective hydrolysis of the H-D peptide bond by SARSCoV-2 was validated using the protease obtained from closely associated viruses, such as MERS-CoV. The same concentration of MERS-CoV viral particles were incubated with FITCAhx-H-d-K(Dabcyl), under the similar condition as SARSCoV-2 as mentioned in Section 2.5, and presented in Fig. 5. There was no significant increase in the fluorescence intensity (less than 20\%) of FITC-Ahx-H-d-K(Dabcyl) in the presence of MERS-CoV virus. The MERS-CoV protease did not cleave the H-d peptide link in the FITC-Ahx-H-d-K(Dabcyl) substrate. The change in the fluorescence intensity was close to the FITC-Ahx-H-d-K(Dabcyl) peptide in the absence of SARS-CoV-2 virus and control peptide, FITC-Ahx-R-h$\mathrm{K}$ (Dabcyl) which confirmed very minimum fluorescence

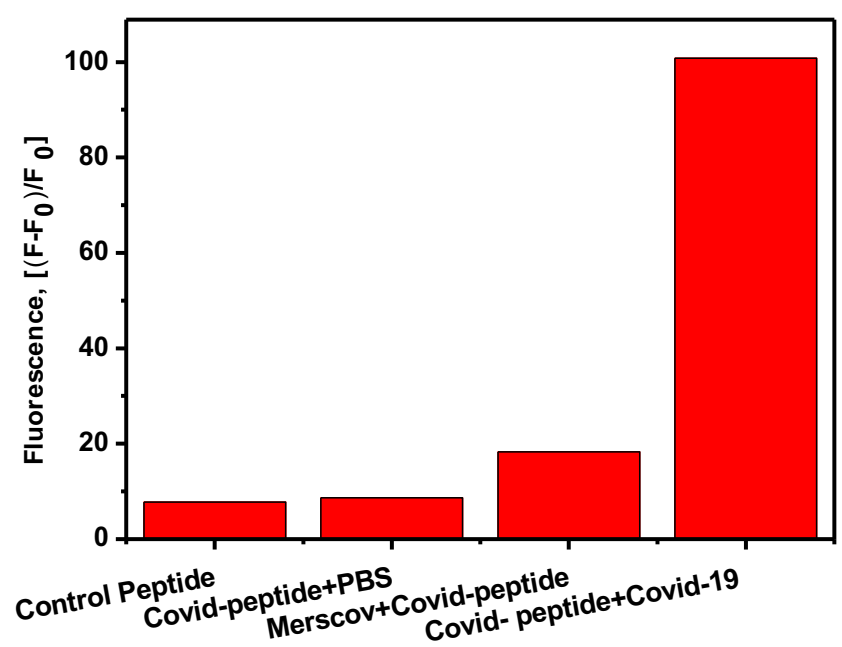

Fig. 5 Changes in the fluorescence of FITC-Ahx-H-d-(Dabcyl) peptide substrate in the presence Covid-19 live virus, Mers-CoV $\left(10^{8} \mathrm{pfu} / \mathrm{mL}\right)$ and PBS buffer after 3 hours of incubation at $37^{\circ} \mathrm{C}$. The samples were excited at $485 \mathrm{~nm}$ and the fluorescence was observed at $535 \mathrm{~nm}$. The standard errors were calculated from the three different measurements 
change in the presence of SARS-CoV-2. The results indicated that the fluorogenic substrate, FITC-Ahx-H-d-K(Dabcyl), is very specific to SARS-CoV-2. Further, it could specifically digest H-D peptide bond in the mixture of other closely associated viral particles. The specific substrate was validated by incubating SARS-CoV-2-positive patient sample with FITCAhx-H-d-K(Dabcyl), and it showed the value of 34,304 pfu/ $\mathrm{mL}$ from the standard calibration plot. The results suggest that FITC-Ahx-H-d-K(Dabcyl) could be used for the diagnosis of SARS-CoV-2-positive samples in bulk in a short time using simple spectrofluorometric measurements.

\section{Conclusions}

We successfully developed a high-throughput peptide screening for the sensitive detection of SARS-CoV-2 as a diagnostic marker. FRET assay has been carried out using fluorogenic dipeptides (L, D, or both amino acids in the dipeptide) with a fluorophore and a quencher at both ends. SARS-CoV-2 protease-specific dipeptide substrates were identified from the quantity of fluorescence signal change. FITC-Ahx-H$\mathrm{d}$-(Dabcyl) fluorogenic dipeptide is considered as the most specific peptide substrate as it showed the highest fluorescence signal in the presence of SARS-CoV-2 protease. The cross-reactivity of the substrate with other closely associated viral proteases such as MERS-CoV has been confirmed from insignificant fluorescence signal change. Clinical patient samples have been used for the validation of the FRET assay, and the values were highly comparable to the standard RT-PCR methods. Therefore, our FRET assay is straightforward, low cost, and highly sensitive for the analysis of clinical patient samples.

Funding The author acknowledges the financial support provided by King Abdulaziz City for Science and Technology (General Directorate for Research \& Innovation Support) (GDRIS) (King Abdulaziz University) to implement. This work is through fast track program for COVID-19 Research Project No. 5-20-01-009-0002.

\section{Declarations}

Conflict of interest This work is protected under USPTO filing No. 17108263.

\section{References}

1. Rothan HA, Byrareddy SN (2020) The epidemiology and pathogenesis of coronavirus disease (COVID-19) outbreak. J Autoimmun 109:102433

2. Hamre D, Procknow JJ (1966) A new virus isolated from the human respiratory tract. Proc Soc Exp Biol Med 121:190-193
3. Chen Y, Chan KH, Kang Y, Chen H, Luk HKH, Poon RWS, Chan JFW, Yuen KY, Xia N, Lau SKP, Woo PCY (2015) A sensitive and specific antigen detection assay for Middle East respiratory syndrome coronavirus. Emerg Microbes Infect 4:e26

4. Williamson EJ, Walker AJ, Bhaskaran K, BaconS BC, Morton CE, Curtis HJ, Mehrkar A, Evans D, Inglesby P, Cockburn J, McDonald HI, MacKenna B, Tomlinson L, Douglas IJ, Rentsch CT, Mathur R, Wong AYS, Grieve R, Harrison D, Forbes H, Schultze A, Croker R, Parry J, Hester F, Harper S, Perera R, Evans SJW, Smeeth L, Goldacre B (2020) Factors associated with COVID-19-related death using OpenSAFELY. Nature 584:430 436

5. Tan WYT, Wong LY, Leo YS, Toh MPHS (2020) Does incubation period of COVID-19 vary with age? A study of epidemiologically linked cases in Singapore. Epidemiol Infect 148:e197

6. Zaim S, Chong JH, Sankaranarayanan V, Harky A (2020) COVID19 and multiorgan response. Curr Probl Cardiol 45:100618

7. Corman VM, Landt O, Kaiser M, Molenkamp R, Meijer A, Chu DKW, Bleicker T, Brünink S, Schneider J, Schmidt ML (2020) Detection of 2019 novel coronavirus (2019-nCoV) by real-time RT-PCR. Eurosurveillance 25:2000045

8. Adhikari SP, Meng S, Wu YJ, Mao YP, Ye RX, Wang QZ, Sun C, Sylvia S, Rozelle S, Raat H (2020) Epidemiology, causes, clinical manifestation and diagnosis, prevention and control of coronavirus disease (COVID-19) during the early outbreak period: a scoping review. Infect Dis Poverty 9(1):1-12

9. Chan WJF, Yip CCY, To KKW, Tang THC, Wong SCY, Leung KH, Fung AYF, Ng ACK, Zou Z, Tsoi HW (2020) Improved molecular diagnosis of COVID-19 by the novel, highly sensitive and specific COVID-19-RdRp/Hel real-time reverse transcriptionPCR assay validated in vitro and with clinical specimens. J Clin Microbiol 58(5)

10. World Health Organization (2020) Coronavirus disease (COVID19) technical guidance: laboratory testing for $2019-\mathrm{nCoV}$ in humans. Switzerland, Geneva

11. Cai X, Chen J, Hu J, Long Q, Deng H, Fan K, Liao P, Liu B, Wu G, Chen Y (2020)A peptide-based magnetic chemiluminescence enzyme immunoassay for serological diagnosis of corona virus disease 2019 (COVID-19), medRxiv

12. Guo L, Ren L, Yang S, Xiao M, Chang D, Yang F, Dela Cruz CS, Wang Y, Wu C, Xiao Y, Zhang L, Han L, Dang S, Xu Y, Yang QW, Xu SY, Zhu HD, Xu YC, Jin Q, Sharma L, Wang L, Wang J (2020) Profiling early humoral response to diagnose novel coronavirus disease (COVID-19). Clin Infect Dis 71:778-785

13. Corman VM, Muller MA, Costabel U, Timm J, Binger T, Meyer B, Kreher P, Lattwein E, Eschbach-Bludau M, Nitsche A, Bleicker T, Landt O, Schweiger B, Drexler JF, Osterhaus AD, Haagmans BL, Dittmer U, Bonin F, Wolff T, Drosten C (2012) Assays for laboratory confirmation of novel human coronavirus (hCoV-EMC) infections. Euro Surveill 17(49) http://www.eurosurveillance.org/ ViewArticle.aspx?ArticleId=20334

14. Udugama B, Kadhiresan P, Kozlowski HN, Malekjahani A, Osborne M, Li VYC, Chen H, Mubareka S, Gubbay JB, Chan WCW (2020) Diagnosing COVID-19: the disease and tools for detection. ACS Nano 14:3822-3835

15. Kustin T, Ling G, Sharabi S, Ram D, Friedman N, Zuckerman N, Bucris ED, Glatman-Freedman A, Stern A, Mandelboim M (2019) A method to identify respiratory virus infections in clinical samples using next-generation sequencing. Sci. Rep 9:2606-2606

16. Keightley MC, Sillekens P, Schippers W, Rinaldo C, George KS (2005) Real-time NASBA detection of SARS-associated coronavirus and comparison with real-time reverse transcription-PCR. J Med Virol 77:602-608

17. Campos EVR, Pereira AES, de Oliveira JL, Carvalho LB, GuilgerCasagrande M, de Lima R, Fraceto LF (2020) How can 
nanotechnology help to combat COVID-19? Opportunities and urgent need. J Nanobiotechnol 18:125

18. Zhu W, Xu M, Chen CZ, Guo H, Shen M, Hu X, Shinn P, KlumppThomas CMichael SG, Zheng W (2020) Identification of SARSCoV-2 3CL protease inhibitors by a quantitative high-throughput screening. ACS Pharmacol Transl Sci 3:1008-1016

19. Rut W, Groborz K, Zhang L, Sun X, Zmudzinski M, Hilgenfeld R, Drag M (2020) Substrate specificity profiling of SARS-CoV-2 Mpro protease provides basis for anti-COVID-19 drug design. Biorxiv. https://doi.org/10.1101/2020.03.07.981928

20. Al-Amri SS, Abbas AT, Siddiq LA, Alghamdi A, Sanki MA, AlMuhanna MK, Alhabbab RY, Azhar EI, Li X, Hashem AM (2017) Immunogenicity of candidate MERS-CoV DNA vaccines based on the spike protein. Sci Rep 7:44875

21. Coleman CM, Frieman MB (2015) Growth and quantification of MERS-CoV infection. Curr Protoc Microbiol 37:15E-2

22. Kaman WE, Voskamp-Visser I, de Jongh DMC, Endtz HP, van Belkum A, Hays JP, Bikker FJ (2013) Evaluation of a D-aminoacid-containing fluorescence resonance energy transfer peptide library for profiling prokaryotic proteases. Anal Biochem 441:38-43

23. Kaman WE, Hulst AG, van Alphen PTW, Roffel S, van der Schans MJ, Merkel T, van Belkum A, Bikker FJ (2011) Peptide-based fluorescence resonance energy transfer protease substrates for the detection and diagnosis of Bacillus species. Anal Chem 83:2511-2517

24. Chinnappan R, Dubé A, Lemay J-F, Lafontaine DA (2013) Fluorescence monitoring of riboswitch transcription regulation using a dual molecular beacon assay. Nucleic Acids Res 41(10): e106-e106

25. Raja C, Ferner J, Dietrich U, Avilov S, Ficheux D, Darlix J-L, de Rocquigny H, Schwalbe H, Mély Y (2006) A tryptophan-rich hexapeptide inhibits nucleic acid destabilization chaperoned by the HIV-1 nucleocapsid protein. Biochemistry 45(30):9254-9265

26. Lam H, Oh DC, Cava F, Takacs CN, Clardy J, de Pedro MA, Waldor MK (2009) D-amino acids govern stationary phase cell wall remodeling in bacteria. Science 325:1552-1555

27. Aliashkevich A, Alvarez L, Cava F (2018) New insights into the mechanisms and biological roles of D-amino acids in complex ecosystems. Front Microbiol 9:683

28. Alhogail S, Suaifan GARY, Zourob M (2016) Rapid colorimetric sensing platform for the detection of Listeria monocytogenes foodborne pathogen. Biosens Bioelectron 86:1061-1066

29. Van de Plassche MAT, Barniol-Xicota M, Verhelst S (2020) Peptidyl acyloxymethyl ketones as activity-based probes for the main protease of SARS-CoV-2. Chembiochem 21:3383-3388. https://doi.org/10.1002/cbic.202000371

30. van Kasteren PB, van der Veer B, van den Brink S, Wijsman L, de Jonge J, van den Brandt A, Molenkamp R, Reusken CBEM, Meijer A (2020) Comparison of commercial RT-PCR diagnostic kits for COVID-19. J Clin Virol 128:104412

31. Seo G, Lee G, Kim MJ, Baek SH, Choi M, Ku KB, Lee CS, Jun S, Park D, Kim HG, Kim SJ, Lee JO, Kim BT, Park EC, Kim SI (2020) Rapid detection of COVID-19 causative virus (SARS$\mathrm{CoV}-2$ ) in human nasopharyngeal swab specimens using fieldeffect transistor-based biosensor. ACS Nano 14:5135-5142

32. Song Y, Song J, Wei X, Huang M, Sun M, Zhu L, Lin B, Shen H, Zhu Z, Yang C (2020) Discovery of aptamers targeting the receptor-binding domain of the SARS-CoV-2 spike glycoprotein. Anal Chem 92:9895-9900

33. Ganguli A, Mostafa A, Berger J, Aydin MY, Sun F, de Ramirez SA, Valera E, Cunningham BT, King WP, Bashir R (2020) Rapid isothermal amplification and portable detection system for SARSCoV-2. Proc Natl Acad Sci 117:22727-22735

34. Varlamov DA, Blagodatskikh KA, Smirnova EV, Kramarov VM, Ignatov KB (2020) Combinations of PCR and isothermal amplification techniques are suitable for fast and sensitive detection of SARS-CoV-2 viral RNA. Front Bioeng Biotechnol 8:1294

35. Ramachandran A, Huyke DA, Sharma E, K. Sahoo M K, Huang C, Banaei N, Pinsky BA, Santiago J G. (2020) Electric field-driven microfluidics for rapid CRISPR-based diagnostics and its application to detection of SARS-CoV-2. PNAS. 117:29518-29525

36. Carter LJ, Garner LV, Smoot JW, Li Y, Zhou Q, Saveson CJ, Sasso JM, Gregg AC, Soares DJ, Beskid TR (2020) JerveySR, Liu C. ACS Cent Sci 6:591-605

37. Eissa S, Zourob M (2021) Development of a low-cost cotton-tipped electrochemical immunosensor for the detection of SARS-CoV-2. Anal Chem 93(3):1826-1833

38. Liu H, Dai E, Xiao R, Zhou Z, Zhang M, Bai Z, Shao Y, Qi K, Tu J, Wang C, Wang S (2021) Development of a SERS-based lateral flow immunoassay for rapid and ultra-sensitive detection of antiSARS-CoV-2 IgM/IgG in clinical samples. Sens Actuators B Chem 329:129196

Publisher's note Springer Nature remains neutral with regard to jurisdictional claims in published maps and institutional affiliations. 